\title{
Lost Puppy: Towards a Playful Intervention for Wandering Dementia Patients
}

\author{
Yacintha Aakster ${ }^{1},{ }^{凶}$ Robby van Delden ${ }^{2[0000-0002-6592-1199]}$, and Stefan \\ Lentelink ${ }^{3}$ \\ ${ }^{1}$ Creative Technology Bachelor, University of Twente, Enschede, the Netherlands \\ yacintha. aakster@gmail.com \\ 2 Human Media Interaction, University of Twente, Enschede, the Netherlands \\ $r$.w.vandelden@utwente.nl \\ 3 Roessingh Research and Development, Enschede, the Netherlands \\ s.lentelink@rrd.nl
}

\begin{abstract}
Many nursing homes for dementia patients struggle with residents that wander towards the exit with the intention of leaving. Several types of interventions have been used to deal with this issue. Unfortunately, many of them are quite forceful, or are unsuitable for the specific context of certain nursing homes. In this paper, we investigate the possibility to using a more playful persuasive intervention. The design itself is in the form of a lost puppy, equipped with several actuators and sensors, that has to be brought 'home', in order to steer residents unknowingly away from the exit. Our first pilot indicated that residents noticed the puppy and showed interest in the device, and might be distracted from the exit. However, the puppy in its current form did not yet lead the residents away from the exit. Based on our contextual analyses, related work, and received feedback, we share our design insights which could be helpful for creating playful interventions for people with dementia.
\end{abstract}

Keywords: Dementia $\cdot$ Wandering $\cdot$ Playful Intervention $\cdot$ Play $\cdot$ Design $\cdot$ Exit - Care Home

\section{Introduction}

Around 47 million people worldwide have dementia [42]. Dementia is an umbrella term for the deterioration of cognitive functions caused by damaging of the cells in the brain and the connections between the cells [2]. The most common type of dementia is Alzheimer's disease [2,42]. The symptoms and characteristics of dementia affect people's everyday functioning and can involve (but are not limited to) memory loss, affected judgement, aggression, loss of willpower, and disorientation of time and place [14,42]. In general, in the early stages of dementia, people are able to remain at home with the help of family caregivers $[23,43]$. When the workload of caregivers becomes too high, the people with dementia often move into a nursing home. Wandering behaviour of people with dementia, especially prevalent for those with Alzheimer's disease, represents a major 
challenge regarding this group [20]. Kleine et al. and Burns et al. indicated that respectively $17.4 \%$ and $18.5 \%$ of their subjects showed wandering behaviour, with subjects with more severe dementia showing higher rates of wandering behaviour $[9,20]$.

Given this high prevalence rate of people with dementia in our society, it is not surprising to see a serious interest of Human-Computer Interaction (HCI) researchers in people with dementia [24]. Joddrell and Astell indicated they found three directions regarding HCI for people with dementia: screening and assessment, addressing activity of daily living (ADL), and providing leisure activities [19]. In this paper we focus on a playful interactive intervention for people with dementia who wander towards the exit with the intention of leaving. The intervention we envision is not aiming for therapeutic goals or cognitive stimulation. Instead, we want to use playful aspects to unknowingly steer people with dementia away from the exit in a more pleasurable way than current alternatives. The case study we present here is for one specific nursing home, where many residents stroll freely through the building. Several of them are in a far developed stage of dementia, and therefore not allowed to leave without supervision. A chip in their shoe prevents the exit door from opening when they stand in front of $i t^{4}$, which can lead to confrontation and confusion. Residents can get upset, cause a scene in the entrance hall, and even become aggressive towards caregivers. This requires the receptionist and caregivers to step in by calming them down and taking them somewhere else.

To address this issue we propose our Lost Puppy concept: a stuffed puppy located near the exit, that is equipped with a motion sensor and several actuators (sound and light) to attract the attention of wandering residents who might go towards the exit. The puppy entices the person to return the puppy to the lost puppy's parent, a non-interactive stuffed dog placed a little further away, intended to lead the resident away from the exit.

In the remainder of this paper we will explain how we came to the design of the Lost Puppy prototype, and share our insights. In Section 2, we start with related work, including other interventions for wandering residents. In Section 3 , we summarise previously identified aspects for addressing the senses that are relevant when designing interactive technology for people with dementia. Section 4 reports on the context of the exit, by conducting a small observational study of the entrance, and several sets of semi-structured interviews with the activity manager, receptionist, and psychologist. In Section 5, we give an overview of the design and concept of the proposed puppy prototype, with which we did a first preliminary evaluation that is reported in Section 6. In Section 7, we discuss some of the weaknesses of our concept, future improvements, and share important design insights. These insights are an important part of the paper, as they point to important aspects and useful directions for the design of interactive technology for people with dementia. And finally, we conclude our work in Section 8 with a short conclusion.

\footnotetext{
${ }^{4}$ A system that is used by more nursing homes, for instance the commercial system 'Door Guardian' [32]
} 
The main contributions of this paper are: (1) to inspire others with our concept to consider a more subtle entertainment perspective than the common forceful devices and interventions to address wandering behaviour (and perhaps related issues); and (2) to provide interesting and useful insights into research and design aspects when working on interactive technology for people with dementia.

\section{Related Work}

For our project we looked at several existing interventions in the literature and those that are commercially available. There are several interventions that help to prevent people with dementia from leaving a nursing home, including preventing doors from opening, delayed opening of doors, hidden doors or controls, signalling an alarm, and surveillance [44]. Alternative ideas we found include a 'phantom bus stop' that makes residents patiently wait for a bus that will never arrive, but might calm down residents and allow caregivers to pick them up at a known location, see [34]. Another method is to use visual deterrents, for instance stickers disguising the door as a book closet, or even less subtle by using yellow signs saying 'STOP', see [36]. Both the signage and door covering stickers were rejected as valid options by the nursing home. A door-sticker with bricks was tried once, but was recognised as covering an exit and subsequently peeled off by a resident.

Interactive technology was seen by the nursing home, and our project team, as a powerful tool that might help to address the wandering problem in different ways. Morrissey et al. indicate that in HCI for people with dementia, technology is often seen as a solution to behavioural decline [24]. For instance, a prompting device using artificial intelligence to help in a routine like washing hands [22], and other forms of assistive technology, including agendas, video calls, or location tracking [19]. Besides technology for activities of daily living (ADL), one can make a distinction between digital leisure activities for pure entertainment and those for cognitive stimulation or therapeutic interventions [19]. Included in the former are creative digital activities such as painting a vase or music making, activities that are (more) difficult to perform without technology [5, $19,30]$. Included in the latter, mainly therapy-related, is research intended to aid current interventions and therapies by introducing additional technology. For example, providing audio and video material using a touch screen interface for reminiscence therapy [11]. An activity for the elderly that can be used for therapy purposes and entertainment is the PARO robot, a robotic baby seal [40]. Similarly in-between is the provision of leisure activities stimulating physical, cognitive, and social aspects in a playful way such as an interactive table projection [3]. This shows that technology is not always 'fixing' behaviour seen in people with dementia, but can also be an entertaining tool to enhance their existing activities.

Similar to the more comprehensive 'nighttime wandering system' of Radziszewski et al., that encouraged people with dementia to go back to bed in a soothing way with lighting and the use of media at night, we are interested in an 
interactive system that can be used during the day to gently steer people in an entertaining and playful way [28]. Nudging interventions (i.e. transparent ways to change behaviour without economic incentives [35]) like these, have been used as a successful way of 'ambient influence' to steer the behaviour of the general public, for instance to get people to use the stairs instead of the elevator [31].

\section{Addressing the Senses of People With Dementia}

When designing for people with dementia, several aspects of (multi-modal) sensemaking should be considered. Several studies suggest this goes beyond only the age-related aspects (see [6] for a more extensive overview). We target several design oriented aspects and address four senses: visual, auditory, olfactory and tactile. We had omitted the sense of taste beforehand as it is beyond the scope of our project.

According to Jakob \& Collier $[16,17]$, the use of visuals can be effective, but is often too much emphasized as a sensory stimulus for people with dementia. Both Habell and Jakob \& Collier mention that people with dementia can have a false visual perception, for instance mistaking a staircase for a waterfall, or a high glare floor surface for a river $[12,17]$. The confusion and decline in visual function is why Jakob \& Collier discourage using shiny objects, moving lights (e.g. a disco ball), and suggest facilitation of slower light intensity changes [17]. The use of visual cues, including signs/icons and objects, can nonetheless be a suitable way to prompt certain behaviour $[5,12]$. Building on his experience as an architect for dementia care centres, Habell suggests that colour coding might be effective, for example giving accessible doors a bold colour [12].

People with dementia might not be able to detect auditory stimuli as well as other people. Hearing loss is a common occurrence in the ageing process. Also, Uhlmann et al. and Lin et al. have found a correlation between hearing loss and the severity of cognitive dysfunction [21,39]. Hearing impairment should therefore be taken into account when using auditory stimuli to attract the attention of people with severe dementia. In terms of auditory stimuli, music has proven to be a useful tool in stimulating people with dementia $[6,30]$. It can be used as a way to comfort people but also allows them to reminisce about earlier times. Spiro indicates that memory for music is special for people with dementia, as it is a complex skill that has been demonstrated to be more persistent than other information abilities [33]. Nair et al. conclude, based on a literature review and their baroque music intervention study (with negative effects), that especially preferred music genres help, rather than music of a general genre [26].

Olfactory deficits are more prominent in people with Alzheimer's disease; deteriorating olfactory discrimination, odour identification, and the olfactory detection threshold [10]. Nevertheless, the use of smells can be beneficial for people with Alzheimer's disease. For example, a small scale aromatherapy study indicated towards improvement of cognitive functioning and some improvement in conceptual understanding [18]. More directly related to HCI research, Gowans et al. speculated that smell will have strong connections with memory functions 
and therefore also suggested the inclusion of smell in their set of (touchscreen operated) reminiscence devices for residents [11].

The sense of touch can be used in a passive and an active way. Passively, to comfort people with dementia and make them feel safe, by providing soft or fluffy materials. And actively, by providing them with objects with which they can interact in engaging ways. For example, sensory cushions are objects that contain different materials for a tactile and multi-faceted experience (vision, smell, hearing, and even taste) [17]. Another distinction can be made whether touch is used instrumentally, as part of a task, or as an expressive form, which is more emotional [6]. We refer the reader to [15] for an overview and several studies on technology addressing social touch and emotional aspects. Touch can be an effective stimulus, but in order to attract people with dementia, and in order to establish interaction, the objects should look inviting and meaningful [17].

\section{Context of the Exit}

The first author did four observations at the location, each lasting about 3 hours and performed during different time frames: $1 \mathrm{x}$ morning, $2 \mathrm{x}$ midday, and $1 \mathrm{x}$ midday/afternoon. Weather conditions may have been of influence. Due to sunny and warm weather, many people (e.g. employees and supervised residents) went outside for a walk or to sit in the sun. On average, about 55 people walked in and out every hour during the observed hours. The entrance hall shows several remarkable points, such as the large contrast in light intensity, see Figure 1. Compared to the bright incoming sunlight, the entrance hall is quite dark. Providing sunlight to the residents is important [12] but residents are also drawn by this. Where the building is well designed for its visitors, who naturally feel where the exit is and where to find information, the design seems less favourable for its wandering residents. The receptionist also plays a role, and can be seen as a multi-sensory stimulus for the residents. The receptionist talks, moves and attracts people, creating an auditory, visual, and social stimulus. In front of the exit, there is a small gift shop. Some residents were observed to be drawn to this shop to look at, and touch, stalled and displayed items (e.g. shiny jewellery and things wrapped in squeaky plastic foil). And lastly, the decor of the entrance hall creates a funnel-like path towards the exit, feeling natural to follow.

\subsection{Interviews}

To get a more personal insight into the behaviour of the residents, beyond a general description of people with dementia, the first author interviewed an activity manager, a receptionist, and a psychologist. A semi-structured interview was used starting with 11-14 questions depending on the expertise of the interviewee. Responses were audio recorded and consent was given for this. We will briefly share the most important findings. 


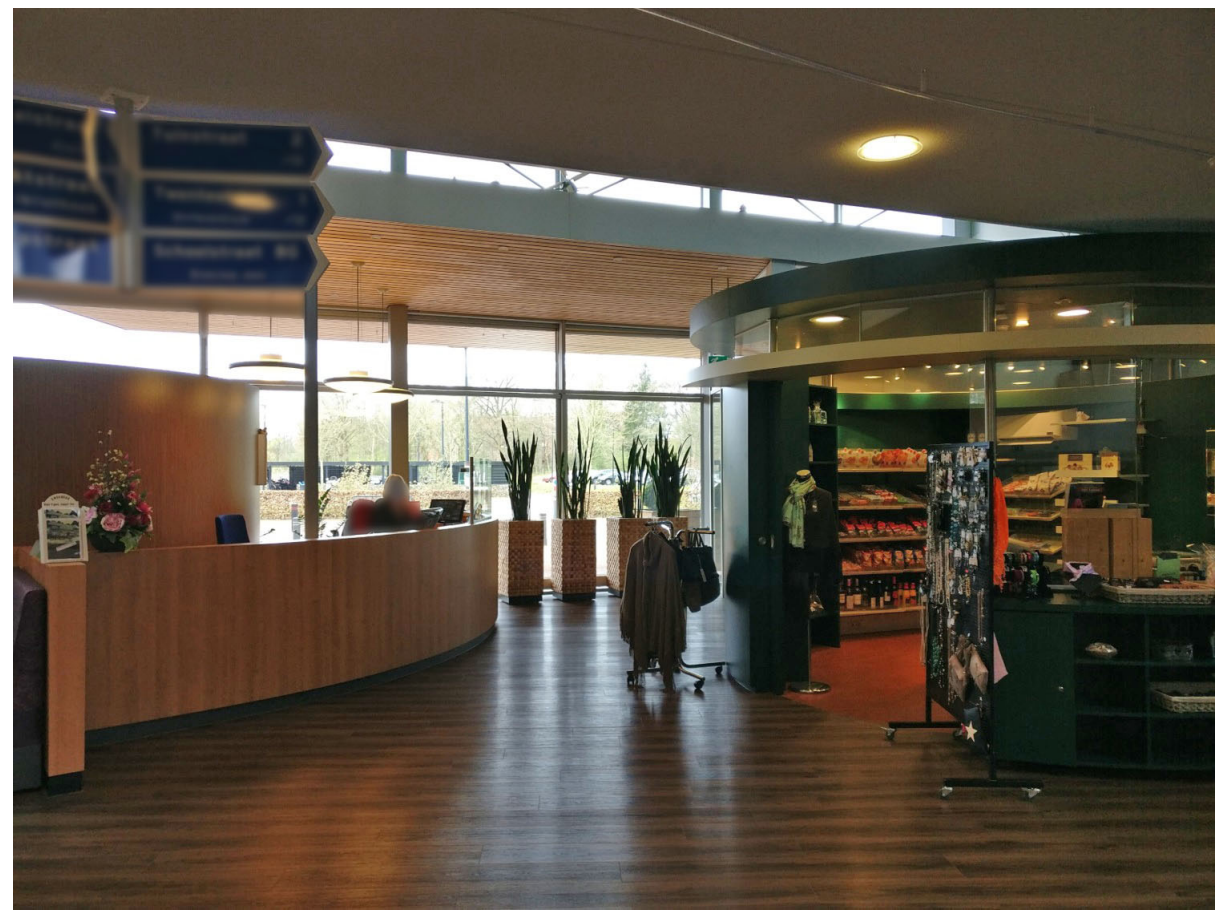

Fig. 1. A shadow/highlight corrected photo of the entrance hall of the nursing home. Notice the funnel effect with attractive light of the exit, the position of the receptionist (blurred for privacy), and the shiny items near the gift shop on the right.

Activity Manager. The activity manager has been working for the nursing home for more than ten years. He organizes and manages activities for the residents with the focus on physical activity and engagement. His goal is to keep residents active and for that reason most activities are related to sports in a playful way.

For giving instructions to the residents, he stated it is important to maintain the order of: 1) having some 'small talk'; 2) showing an explanatory photo or picture; and 3) performing the activity. In other words, explain, demonstrate, and then let them try it out themselves. 'Only words are not enough.' He also mentioned that the best way to reach residents, for him personally, is to use humour. Making residents laugh makes them comfortable and relaxed, and makes them more willing to participate. The activity manager tried incorporating the Kinect into activities about 3 years ago, but the relation between physical actions and the screen were not understood by the residents. However, considering new kinds of technologies, he continued that 'the people coming into the nursing home nowadays are a bit more familiar with it'. 
Receptionist. The interviewed receptionist has been working at the nursing home for eight years. Her primary task is to be a receptionist, but an important part of her job is also to interact with the residents that come to her desk, or the exit.

She indicated that residents who go to the exit most often come just after the lunch, when the sun is shining right on the glass around the exit, and when the employees change shift. She mentioned that the latter is an important stimulus for residents, because employees gather at the desk. Residents sometimes end up at the exit by accident as a result of their wandering, and sometimes because they planned to. It is the hardest to distract or change the mind of the latter group of residents. It is also more common that this group of residents get upset or aggressive. To lead residents away from the exit, the receptionist uses a personal approach (including the use of names) which she feels is the most effective.

Psychologist. The interviewed psychologist has been working in the field of elderly care for over eight years. She does neurological research on dementia and is involved with the residents. She decides, based on a person's stage of dementia, symptoms, and behaviour, what the best care for this person would be.

When asked about the utility and applicability of sensory stimuli in guiding residents away from the exit, the psychologist stated she could imagine it being useful. People with dementia often have difficulties with focussing and are easily distracted and attracted by stimuli. She strongly believes that providing a counter stimulus that is more attractive than the exit could help in solving this problem. The psychologist explained that the time it takes to process sensory stimuli is different for people with dementia as opposed to healthy people. A person with dementia needs more time to process sensory stimuli, and therefore it is important to provide these stimuli a little bit longer than normal. She experiences dementia as a progressive disease that slowly takes away memories, starting with the last learned things and making its way back through time. People with dementia have a different perception of the world because they have lost memory of the things learned recently. Mentally they could be back 40 years, making it harder to incorporate newer technologies, as was indicated by the activity manager as well. The psychologist feels that a combination of both an autonomous distraction from a technological intervention and a personal, human, approach is preferable and probably most effective.

\section{Design of the Proposed Puppy Prototype}

\subsection{A Stuffed Animal}

Taking into account the insights from the literature, observations, and interviews mentioned above, the first author created 50 ideas for concepts through individual brainstorming. All ideas were then evaluated mainly by the first author, in four successive phases. Input from two occupational therapists was used as input 
for phases three and four. In the first phase, twelve seemingly unrealistic or infeasible ideas were filtered out. In the second phase, the remaining ideas were scored on the following principles: feasibility; innovativeness; comfortability; distractability/attractiveness; fit with the environment; and safety. The principles were scored between one to five points for each concept. The ideas that scored 20 points or higher, with a maximum of 30 points, were then elaborated upon giving concept descriptions. In the third phase the resulting top 12 of concepts were subsequently pitched to the two collaborating occupational therapists. During this third phase, the therapists indicated the general expected effectiveness of the concepts, they did this based on their expertise in the field of dementia and experience with residents of the nursing home. The gathered feedback was used to come to a top three of concepts. In the fourth phase these three concepts were assessed by weighing of pros and cons by the first author. The Lost Puppy concept was selected because of its expected effectiveness (as indicated in phase three by the therapists) in combination with its soft and playful character.

The Lost Puppy concept consists of a stuffed animal in the shape of a puppy, paw prints on the floor that indicate the direction of the goal, and a larger stuffed animal in the shape of a dog to represent the puppy's parent. The concept is supported by a storyline about the puppy being lost and needing to be brought back home, barking for attention. This home is located elsewhere in the residence where the parent dog is laying in a dog basket. The route from the puppy in the entrance hall to its parent dog is indicated by small paw prints on the floor. By giving residents the task to bring back the puppy, an interaction can be established that will engage them for a longer time. By performing this task, residents are unknowingly distracted from their own goal (i.e. going to the exit) and being led away from the stimuli that could re-attract them towards the exit. See Figure 2, for an overview of the intervention.

The choice of animal in this concept was a dog, based on people's familiarity with dogs and the importance of recognition in design for people with dementia, see $[1,5,17,26]$. Also, a dog was a good fit for the storyline. There is however, a possibility that people have a fear of dogs. For that reason, a puppy was used instead of a mature dog because they are considered to be more approachable. Puppies also possess certain physical properties (infantile) that can stimulate caregiving in people [7]. Giving care to a vulnerable puppy can raise a feeling of responsibility in residents, and therewith stimulate them mentally. In another study, such a nurturing aspect with tangible properties in playful activities has also been indicated by proxies of residents as bringing pleasure [37]. Succeeding in reuniting the puppy with its parent can provide a feeling of complaisance and adds a clear goal.

\subsection{Location}

The location of the puppy was decided based on a small set of tests. For the tests, a stuffed animal in the shape of a small dog was placed at different locations in the entrance hall of the nursing home. For the course of an hour for each location, the first author observed whether the dog would be spotted or not 


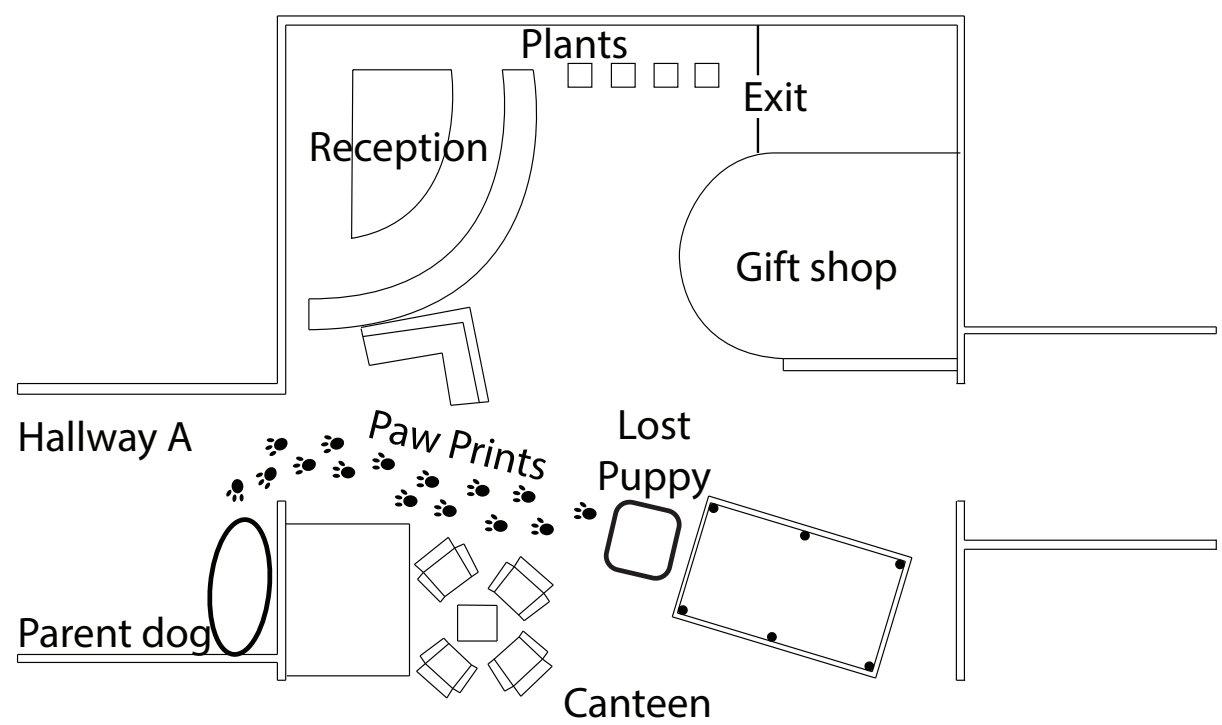

Fig. 2. A schematic overview of the exit location of the nursing home with the Lost Puppy concept.

by the resident. The most reactions were witnessed when the dog was put on top of a pool table that had a black cover on it. At this location there was a large contrast between the black canvas and the light coloured fluffy animal. Therefore, we decided to use this location. However, to allow the pool table to be used, we used a moveable cart in front of the table on which the puppy could sit.

\subsection{Technical Implementation}

The implemented system consisted of a commercially available stuffed puppy of around 35 centimetres long, 15 centimetres wide, and 15 centimetres in height, see Figure 3. To keep the dog light enough and better resistant to being accidentally dropped, the speakers were not embedded in the puppy. Instead, they were included in a moveable pedestal on which the puppy was placed. The pedestal also included a semi-transparent window with a Philips Hue colour changing LED lamp behind it to strengthen the visual attractiveness. Also a note was added around the puppy's neck prompting to bring it back home . A passive infrared motion sensor was connected to an Arduino to trigger a randomly selected barking sound when motion was detected. Five different barking sounds were included, ranging from more whiny to excited barking. Paw prints were placed on the floor to prompt the direction to walk to, the location of the non-interactive 'parent dog' positioned around the corner, as indicated in Figure 2. 


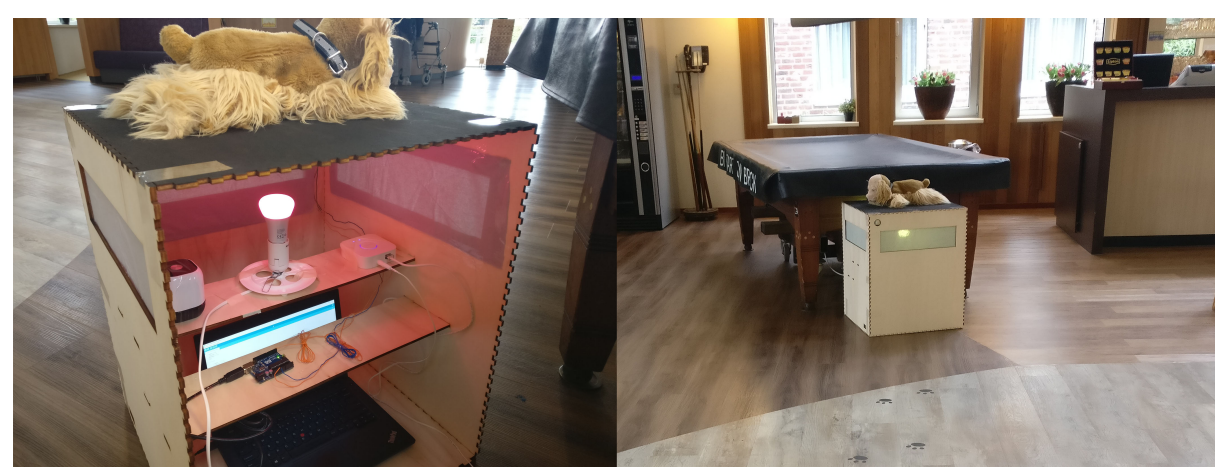

Fig. 3. Two photos of the implementation of the Lost Puppy concept. On the left, the puppy and motion sensor are placed on a pedestal with inside the Philips Hue with control interface, speaker, and laptop running the software. On the right, the installation can be viewed as a resident would see it when walking through the entrance hall.

\section{Pilot Evaluation}

The goal of the pilot evaluation was to gain insight into how effective the intervention is and to indicate whether it is indeed possible to divert the attention of residents away from the exit with such an intervention. In order to gain insights into the effectiveness of the elements of the intervention (i.e. puppy, paws and parent dog, note, and active involvement of the receptionist), starting with the puppy we added one element every 90 minutes (while including the previously added elements). In total, the observations were performed over the course of seven hours. The exact numbers of interactions observed were not kept because observations were performed from several points of view and the exit was not always within sight (e.g. when observing interactions with the parent dog). People with dementia are a rather vulnerable user group, and therefore we worked in close cooperation with the nursing home to perform the user test. We started with a test of one hour to discover any major flaws and to be sure that no dangerous situations could occur during testing.

Puppy. Residents in the entrance hall did notice the puppy and some approached it. One resident walked by the puppy, and said to the puppy when it started barking 'It's going to be fine'. Later that same resident walked by again and responded to the puppy's barking by saying 'Calm, calm'. The resident stood next to the puppy for a couple of minutes but did not pet or pick up the puppy. There was one resident, usually quite active around the exit and regularly attempting to leave, who asked how the system actually worked, even being interested in the technical details. She did not think the puppy was real, but did pet the puppy and seemed to be amused by the whole system for quite a while. 
In the facility there were several groups of residents with visitors. In one group, a visitor suggested to approach the puppy. The group seemed to be very amused and delighted by the puppy and its barking, and one of the residents left a little flower with the puppy. Usually the visitors reacted with the most enthusiasm to the puppy and encouraged the residents to approach and pet the puppy. This created a social situation in which the puppy was central. They would stand around the puppy, listen to its barking, and some also petted the puppy.

There was one resident later that day who came to the exit with the intention of leaving. When the receptionist suggested to go to the puppy, the resident responded saying the puppy on top of the box was fake but that there was a dog inside of that box, because it could be recognised that the barking came from there. However, the resident did not care to seek this out. She did continue to have a conversation with the receptionist and then decided to sit down. There were also residents that passed by the puppy without noticing it, or its barking. It slowly became busier in the entrance hall because lunch hour was approaching, which sometimes made it difficult to hear the barking. The receptionist therefore suggested to turn up the volume.

Paws and Parent Dog. The paw prints seemed to help to gain attention from residents. Residents stopped to look at them and made comments such as how funny'. One resident in the entrance hall had already noticed the puppy, and also noticed the paw prints. He even followed the paw prints for a little while, but not far enough to spot the 'parent dog'. There were also residents coming from the location of the parent dog, walking towards the entrance hall. These residents walked past the parent dog but almost all residents did seem to notice it. Most of these residents responded by smiling, and some reacted verbally both in a group setting and when alone. It did not result in any physical interaction with the dog.

Note. The prompting note 'Lost: bring me to Hallway A', was not noticed by many residents. There was one resident who noticed the note by herself, but she did not engage in further interaction. The visitors that accompanied the residents did notice the note, but they also seemed to lack the confidence to pick up or take the puppy. Therefore, they did not encourage the residents to do so either.

Active involvement of the Receptionist. The receptionist was instructed to point residents towards the puppy when they came to the exit, and explain that the puppy was lost and had to be brought back to the 'parent dog'. With the receptionist's assistance, residents seemed to approach the puppy more often than without her encouragement. Some residents walked up to the puppy upon her instructions, but on arrival they would look at it and walk away again. Some residents did not immediately follow up on the receptionist's instructions, but 
when she physically guided them they would follow her. The residents did not show interest in bringing the dog to Hallway A, and said things such as 'I have to pee first' or 'No, it is dead', without being really bothered by that latter possibly shocking interpretation. The receptionist did state that the puppy provided a natural topic to talk about with residents and that she did not feel uncomfortable to do so. She did not see a change in the behaviour of the residents during the tests, as she believes that most of the residents are too 'faded' to be able to grasp the idea of a lost puppy. She noticed that residents refused to step on the paw prints and therefore (ironically) even suggested to place them directly in front of the exit.

\section{Discussion}

In this work, we created a playful interactive intervention to address the issue of wandering in people with dementia in a nursing home. Our pilot evaluation was very exploratory and tested for only one day. Therefore, long term testing is needed to gain more insight into the interaction and effectiveness of its elements. Such evaluations could also use more quantitative measures with a fairer comparative research methodology (e.g. comparing identical time slots).

Nonetheless, the current study showed that the Lost Puppy concept can attract residents walking through the entrance hall. When noticed, the Lost Puppy system also seems to have a general positive effect on the residents and the atmosphere in the entrance hall. However, the intervention does not succeed in drawing the attention of residents for a long time. Residents did not complete the suggested task of bringing the puppy to its parent. The intervention did not prevent residents who purposefully wanted to leave from going to the exit either. For the residents who did not end up at the exit during testing, we can only speculate if they were steered away by the intervention or by something else. In this discussion we suggest improvements for the current prototype in order to make the Lost Puppy a more suitable intervention. Furthermore, in the subsequent paragraphs we will share more general insights for designing such a playful intervention for people with dementia.

\subsection{Improve the Setting, Sensors, Sounds, and Stimuli of the Puppy}

Similar to the importance of the physical layout of the entrance, the location and setting of the Lost Puppy are also essential. Currently the puppy was placed on top a large pedestal in the entrance hall. To some people this made the puppy less approachable, as if the puppy was exhibited and they were not allowed to touch it. Therefore, it is recommended to place the puppy in a location that is perceived more accessible. Another point regarding the setting is that if the puppy is actually brought to the parent, the puppy in the entrance hall needs to be replaced. We recommend that the receptionist keeps a small storage of puppies nearby, to refill the Lost Puppy spot, and occasionally collects the puppies from 
the parent somewhere nearby. The receptionist present during the evaluation was more than willing to do this.

The receptionists and caregivers at work during the testing indicated that the sounds were sometimes too soft when there was noise in the entrance hall. Therefore, it is recommended to provide the receptionist with the possibility to change settings of the installation, including changing the volume of the barking sounds and to mute it when there is for example a performance (e.g. a children's choir) in the hall.

Currently, the stimuli of the puppy were not sufficing to go beyond attracting the residents. Therefore, we recommend as a first attempt to include more stimuli. For example, the puppy should, similar to the PARO robot, move its head and tail [40]. This could make it appear more natural and, combined with other improvements (e.g. adaptive (barking) sounds), persuade the residents to initiate more action, hold their attention during execution, and finally succeed to bring the puppy to the parent steering the residents away from the exit.

\subsection{Design for Dementia Insights: Towards Guidelines}

The current study does not yet allow us to establish strong guidelines, but sharing our first insights might help others. We based our insights on related work, interviews, and feedback from residents. And we welcome future research to test, strengthen, and add more of these insights. Several of the insights extend the work of Jakob and Collier regarding design principles and recommendations for multisensory environments (MSEs) ${ }^{5}$.

Getting Informed. Regarding the design process we first reiterate that it is important to include people with dementia (and people close to them) in the process, and to educate oneself regarding dementia [19,38]. This can also include education about more specific elements, in this case wandering behaviour and analysis of the exit situation. In this particular study, we leaned in the beginning and concept generation more on the proxies of the residents (i.e. the activity manager, psychologist, receptionist, and occupational therapists), than on direct interactions with the residents. It might have been beneficial to also include more family members. In our opinion the goal of the intervention, unknowingly prevent the residents from leaving, could have been a too confronting topic to incorporate residents as full design partners. For the informal location test and pilot study we did use both the verbal and non-verbal feedback of the residents as much as possible. For insights into proper participatory design and co-design for people with dementia we refer to [13] and [38].

\footnotetext{
${ }^{5}$ The principles consisted of: feeling comfortable and safe; meaningful and familiar; multisensory experience; stimulation and relaxation; control and interaction; and age appropriate and usable. These were accompanied with 3 design recommendations: using textiles and soft materials (encouraging playful engagement), lighting and lights (gradual changes), and low-tech vs high-tech (multi-modal but preventing a technical appearance by using familiar elements). We refer the interested reader to [17] or their more informal and extensive guidebook [16] for a complete description.
} 
Over-stimulation. It is important to prevent over-stimulation for this target group. Too much sensory stimuli can be easily overwhelming. As described by Reisberg et al.'s Stage 3 - mild cognitive decline scale, affected concentration can become apparent for people with dementia [29]. This supports the advice for multi-sensory rooms for residents with dementia by Jakob \& Collier, to declutter spaces and create visual focus points [16]. For example, guiding visual focus with lights that gradually change colour (e.g. with a Philips Hue or light path of LEDs [28]), with moving parts (e.g. moving limbs of a seal [40]), or by increasing contrast (e.g. as seen in the informal location test by choosing the proper background) ${ }^{6}$.

Goals and Prompts. Another aspect is to include clear goals during the entire interaction [1] with the use of appropriate prompts during the interaction. Clear goals can also be intuitive goals. For example, showing, in a virtual environment, dots of paint in combination with a blank vase can be enough to trigger residents to start painting [5]. In our intervention we included a clear goal, but it might not have been clear during the entire interaction. Emphasizing for instance the paws with additional gradually changing lights might have helped to make the goal clearer, and could have prompted the users. The written prompt 'to return the puppy' to its parent's location was not successful. Even auditory prompts (not given by a person) might be ignored when they are hard to understand. Another consideration is that it can also be speculated that prompts using synthetic speech might be harder to understand than naturally spoken text, especially for people with dementia [1]. In the prompts given by a system, it is also important that it is clear what to do, but in the meanwhile preventing that residents become insecure about whether they are capable to perform the task [30]. More extensive, simple and short but very specific, preferably multi-modal prompts seem to be needed.

Personal Approach. In general it helps to use tailoring and personalization to persuade people [27]. For our target group this could for instance be done by using the residents name for prompting (as was mentioned in an interview with the receptionist), adapting content to personal preferences (e.g. changing to preferred music although this might be logistically problematic [26]), or triggering remaining memories with certain topics [12, 41]. However, considering privacy-security reasons, and considering that 'a significant number of subjects' got upset when personal pictures were included (they could not remember the depicted moments or persons), Gowans et al. argue against the use of such personalization [11]. Therefore, it is important to consider what type of personalisation is used and if the benefits outweigh its consequences.

\footnotetext{
${ }^{6}$ Shiny objects were observed to attract attention at the gift shop near the exit, and reflective surfaces can be fascinating for some individuals [38]. However, shiny object/glare/reflections could be misinterpreted by residents and be detrimental and confusing $[6,17]$.
} 
Recall. In the design clearly link to recognisable and/or familiar elements $[1,5,17,26]$. As people with dementia in general have severe trouble learning new skills or memorising new procedures, the interaction should build on recognisable and intuitive elements. Furthermore, according to Jakob and Collier, using familiar elements can help to make users feel safe [17]. One way to address this familiarity is using technology to mimic animals, like the PARO robotic seal [40]. It seems that this could trigger the caregiving tendencies indicated for real human-animal interaction, especially when using infantile characteristics which is known as the baby schema [7].

Incorporating Sounds. When applicable, make sounds originate actually from the object itself, or at least incorporate the origin of sounds into the design. It might be hard for residents to make the cognitive link between the object and sounds playing from a distance. This link was previously also suggested by Gowans et al. [11]. Even though the speaker was really close, the difference in location was noticed for our puppy by one resident, leading her to conclude the puppy was fake. Furthermore, considering the high occurrence of hearing problems of people with Alzheimer's disease [39], it could be useful to have adjustable audio. The audio should be audible but not too loud, preventing discomfort and over-stimulation of others.

Humour, Fun, and Laughter. If possible in a suitable manner, try to incorporate humour into the system. Several sources mention stimulation of humour or laughter for residents $[4,12,23]$. Our current design already triggered some smiling, laughs and giggles, as well as remarks as 'how funny' and 'look at that'. The interviewed activity manager also mentioned humour as an important feature to reach residents.

Social Interaction. Embrace the attractive value, the effect that 'people attract people', and incorporate facilitation of the social interactions it can trigger. In other words: use the interactive piece as a 'honey pot' [8]. This 'enticing honey-pot' effect [8] has been proven to exist for embodied play experiences in public spaces [25]. As was indicated in the interview, it also seemed that residents were attracted when employees gathered. Furthermore, a demonstration effect was seen where people (mostly family visitors) would talk about the puppy to the residents and even pet it. Related to this we also advise to consider the use of the system as 'conversation piece' as this might support and stimulate social interaction. This was seen here, as well as suggested for other systems for people with dementia $[38,41]$, and in a general population interacting in public spaces [25].

Age Appropriate Design. Together with staff and residents we identified that there could be a thin line between offering a too childish toy and providing an effective steering interactive intervention. A too childish looking system might 
make residents less willing to use it, as was also demonstrated in another study by one participant leaving a music activity because it looked too childish [30]. This perfectly fits with Jakob and Collien's principle to make it age appropriate and usable [17]. We follow them and others in the advice to address people with dementia with respect, taking them for full, addressing what they can while taking into account the things they have trouble with, their peculiarities, and the individual differences $[17,23,24,41]$. Perhaps targeting/picking the proper play experiences according to the stage of dementia could be a small help herein $[4]$.

To emphasize the importance of providing pleasurable interactions for people with dementia using an appropriate multi-modal interaction, fitting the person's preferences, we end this discussion with this inspiring quote:

'The emphasis in descriptions of the course of the syndrome tends to be on long-term degeneration. Nevertheless, in a favourable environment and with care based on respect and love, people with dementia can, in spite of their limitations, laugh, dance, sing and cry, mean something special to someone else, care for a fellow resident, and enjoy good food, beautiful music and pleasant smells.' [23, p4]

\section{Conclusion}

This study focused on developing an interactive playful intervention to steer people with dementia living in a nursing home unknowingly away from the exit. Our proposed Lost Puppy concept has not yet succeeded in achieving its goal. However, our study, building on the research of others, resulted in several insights into the design for people with dementia regarding this subject. We believe that these insights can inspire and support future initiatives towards subtle playful interactive interventions for people with dementia.

Acknowledgements. This study was part of a bachelor assignment and is the result of a cooperation between the University of Twente (Human Media Interaction research group), Roessingh Research and Development (Telemedicine Group), and Liberein (location Bruggerbosch). We would like to thank all staff members from Bruggerbosch who provide 24/7 residential care for people with dementia, and in particular Nienke de Haan and Manon Scheffer for the time they invested and their valuable insights. And finally, we would like to thank Dennis Reidsma, Lynn Packwood, and Monique Tabak, and our other colleagues involved in this project for their valuable input. This project was indirectly sponsored as part of a collaborative effort between Roessingh Research and Development and Liberein (location Bruggerbosch). 


\section{References}

1. Alm, N., Astell, A., Gowans, G., Dye, R., Ellis, M., Vaughan, P., Newell, A.F.: An interactive entertainment system usable by elderly people with dementia. In: Stephanidis, C. (ed.) Universal Access in Human-Computer Interaction. Ambient Interaction: 4th International Conference on Universal Access in Human-Computer Interaction, UAHCI 2007 Held as Part of HCI International. pp. 617-623. Springer Berlin Heidelberg, Berlin, Heidelberg (2007)

2. Alzheimer's Association: What is dementia? http://www.alz.org/ what-is-dementia.asp, last accessed 2017/7/29

3. Anderiesen, H.: Playful Design for Activation: Co-designing serious games for people with moderate to severe dementia to reduce apathy. Ph.D. thesis, TU Delft, Delft, The Netherlands (2017), Chapter: Intermezzo: Active Cues Tovertafel - a product description

4. Anderiesen, H., Scherder, E., Goossens, R., Visch, V., Eggermont, L.: Play experiences for people with alzheimers disease. International Journal of Design $9(2)$ (2015)

5. Astell, A., Alm, N., Dye, R., Gowans, G., Vaughan, P., Ellis, M.: Digital video games for older adults with cognitive impairment. In: Miesenberger, K., Fels, D., Archambault, D., Peňáz, P., Zagler, W. (eds.) Computers Helping People with Special Needs: 14th International Conference. pp. 264-271. ICCHP '14, Springer International Publishing, Cham (2014)

6. Behrman, S., Chouliaras, L., Ebmeier, K.: Considering the senses in the diagnosis and management of dementia. Maturitas 77(4), 305-3-310 (2014)

7. Borgi, M., Cirulli, F.: Pet face: Mechanisms underlying human-animal relationships. Frontiers in Psychology 7(MAR), 298:1-11 (2016)

8. Brignull, H., Rogers, Y.: Enticing people to interact with large public displays in public spaces. In: Rauterberg, G.W.M., Menozzi, M., Wesson, J. (eds.) Proceedings of the IFIP International Conference on Human-Computer Interaction. pp. 17-24. INTERACT '03, IOS Press (2003)

9. Burns, A., Jacoby, R., Levy, R.: Psychiatric phenomena in alzheimer's disease. iv: Disorders of behaviour. Br J Psychiatry 157, 86-94 (1990)

10. Djordjevic, J., Jones-Gotman, M., Sousa, K.D., Chertkow, H.: Olfaction in patients with mild cognitive impairment and alzheimer's disease. Neurobiology of Aging 29(5), $693-706$ (2008)

11. Gowans, G., Campbell, J., Alm, N., Dye, R., Astell, A., Ellis, M.: Designing a multimedia conversation aid for reminiscence therapy in dementia care environments. In: CHI '04 Extended Abstracts on Human Factors in Computing Systems. pp. 825-836. ACM, New York, NY, USA (2004)

12. Habell, M.: Specialised design for dementia. Perspectives in Public Health 133(3), 151-157 (2013)

13. Hendriks, N., Truyen, F., Duval, E.: Designing with dementia: Guidelines for participatory design together with persons with dementia. In: Kotzé, P., Marsden, G., Lindgaard, G., Wesson, J., Winckler, M. (eds.) Human-Computer Interaction - INTERACT 2013. pp. 649-666. Springer Berlin Heidelberg, Berlin, Heidelberg (2013)

14. Holzer, C., Warshaw, G.: Clues to early alzheimer dementia in the outpatient setting. Arch Fam Med. 9(10), 1066-1070 (2000)

15. Huisman, G.: Social touch technology: extending the reach of social touch through haptic technology. Ph.D. thesis, Universiteit Twente, Enschede, The Netherlands (2017) 
16. Jakob, A., Collier, L.: A guide book: How to make a Sensory Room for people living with dementia. Kingston University, London, England (2014), http://fada.kingston.ac.uk/de/MSE_design_in_dementia_care/doc/How\% 20 to $\% 20$ make $\% 20$ a $\% 20$ Sensory $\% 20$ Room $\% 20$ f or $\% 20$ people $\% 20$ with $\% 20$ dementia.pdf, unpublished manuscript

17. Jakob, A., Collier, L.: Sensory enrichment for people living with dementia: increasing the benefits of multisensory environments in dementia care through design. Design for Health 1(1), 115-133 (2017)

18. Jimbo, D., Kimura, Y., an Masashi Inoue, M.T., Urakami, K.: Effect of aromatherapy on patients with alzheimer's disease. Psychogeriatrics 9(4), 173 - 179 (2009)

19. Joddrell, P., Astell, J.A.: Studies involving people with dementia and touchscreen technology: A literature review. JMIR Rehabil Assist Technol 3(2), e10 (2016)

20. Klein, D.A., Steinberg, M., Galik, E., Steele, C., Sheppard, J.M., Warren, A., Rosenblatt, A., Lyketsos, C.G.: Wandering behaviour in community-residing persons with dementia. International Journal of Geriatric Psychiatry 14(4), 272-279 (1999)

21. Lin, F.R., Yaffe, K., Xia, J., Xue, Q.L., Harris, T.B., Purchase-Helzner, E., ..., Simonsick, E.M.: Hearing loss and cognitive decline in older adults. JAMA Internal Medicine 173(4), 293-299 (2013)

22. Mihailidis, A., Boger, J., Canido, M., Hoey, J.: The use of an intelligent prompting system for people with dementia. interactions 14(4), 34-37 (2007)

23. Ministry of Health, Welfare and Sport, Zorgverzekeraars Nederland [Health insurance Companies Netherlands], Alzheimer Nederland, ActiZ: Guideline for integrated dementia care [excerpt] (2009), http://ec.europa.eu/social/ BlobServlet?docId=8275, last accessed 2017/10/18

24. Morrissey, K., Lazar, A., Boger, J., Toombs, A.: HCIxDementia workshop: The role of technology and design in dementia. In: Proceedings of the 2016 CHI Conference Extended Abstracts on Human Factors in Computing Systems. pp. 484-491. ACM, New York, NY, USA (2017)

25. Müller, C., Wan, L., Wulf, V.: Dealing with wandering in institutional care: Exploring the field. In: Proceedings of the 7th International Conference on Pervasive Computing Technologies for Healthcare. pp. 101-104. PervasiveHealth '13, ICST (Institute for Computer Sciences, Social-Informatics and Telecommunications Engineering), Brussels, Belgium (2013)

26. Nair, B.R., Browne, W., Marley, J., Heim, C.: Music and dementia. Degenerative Neurological and Neuromuscular Disease 3, 47-51 (2013)

27. Oinas-Kukkonen, H., Harjumaa, M.: Persuasive systems design: Key issues, process model, and system features. Communications of the Association for Information Systems 24(28), 485-500 (2009)

28. Radziszewski, R., Ngankam, H., Pigot, H., Grégoire, V., Lorrain, D., Giroux, S.: An ambient assisted living nighttime wandering system for elderly. In: Proceedings of the 18th International Conference on Information Integration and Web-based Applications and Services. pp. 368-374. iiWAS '16, ACM, New York, NY, USA (2016)

29. Reisberg, B., Ferris, S.H., de Leon, M.J., Crook, T.: The global deterioration scale for assessment of primary degenerative dementia. The American journal of psychiatry $9(3), 1136-1139$ (1982)

30. Riley, P., Alm, N., Newell, A.: An interactive tool to promote musical creativity in people with dementia. Computers in Human Behavior 25(3), 599-608 (2009), including the Special Issue: Enabling elderly users to create and share self authored multimedia content 
31. Rogers, Y., Hazlewood, W.R., Marshall, P., Dalton, N., Hertrich, S.: Ambient influence: Can twinkly lights lure and abstract representations trigger behavioral change? In: Proceedings of the 12th ACM International Conference on Ubiquitous Computing. pp. 261-270. ACM, New York, NY, USA (2010)

32. Secure Care: Wander management solutions. http://www. securecare.com/hubfs/ Brochures/DG_Brochure.pdf?t=1495550876843, last accessed 2017/10/18

33. Spiro, N.: Music and dementia: observing effects and searching for underlying theories. Aging \& Mental Health 14(8), 891-899 (2010)

34. Taylor, K.: The phantom bus stop. Free online journal Hektoen International - A Journal of Medical Humanities 6 (4), http://hekint.org/ the-phantom-bus-stop/ (2014), last accessed 2017/10/18

35. Thaler, R., Sunstein, C.: Nudge: Improving Decisions about Health, Wealth, and Happiness. Yale University Press (2008)

36. The Alzheimer's store: Wandering. http://www.alzstore.com/ alzheimers-dementia-wandering-s/1828.htm, last accessed 2017/10/18

37. Treadaway, C., Fennell, J., Kenning, G., Prytherch, D., Walters, A.: Designing for wellbeing in late stage dementia. In: Proceedings of the Third International Conference Exploring the Multi-Dimensions of Well-Being;Co-Creating Pathways to Well-Being. pp. 126-129 (2016)

38. Treadaway, C., Kenning, G.: Sensor e-textiles: person centered co-design for people with late stage dementia. Working with Older People 20(2), 76-85 (2016)

39. Uhlmann, R.F., Larson, E.B., Rees, T.S., Koepsell, T.D., Duckert, L.G.: Relationship of hearing impairment to dementia and cognitive dysfunction in older adults. JAMA 261(13), 1916-1919 (1989)

40. Wada, K., Shibata, T., Saito, T., Tanie, K.: Effects of robot-assisted activity for elderly people and nurses at a day service center. Proceedings of the IEEE 92(11), 1780-1788 (2004)

41. Wallace, J., Thieme, A., Wood, G., Schofield, G., Olivier, P.: Enabling self, intimacy and a sense of home in dementia: An enquiry into design in a hospital setting. In: Proceedings of the SIGCHI Conference on Human Factors in Computing Systems. pp. 2629-2638. CHI '12, ACM, New York, NY, USA (2012)

42. World Health Organization: Fact sheet dementia (2017), http://www.who.int/ mediacentre/factsheets/fs362/en/, last accessed 2017/10/18

43. Yaffe, K., Fox, P., Newcomer, R., Sands, L., Lindquists, K., Dane, K., Covinsky, K.E.: Patient and caregiver characteristics and nursing home placement in patients with dementia. JAMA 287(16), 2090-2097 (2002)

44. Zeisel, J., Hyde, J., Levkoff, S.: Best practices: An environment behavior (e-b) model for alzheimer special care units. American Journal of Alzheimer's Care and Related Disorders \& Research 9(2), 4-21 (1994) 